48.41]). M1 and M2 did not have a significant effect on the requirement for PT.

Conclusion Massage therapy could be an effective adjuvant to PT in order to reduce the PT duration. However, it did not reduce the requirement for PT.

\section{P485 ANNULAR SKIN LESIONS IN A NEONATE - TRICHOPHYTON SOUDANENSE}

${ }^{1}$ Abhidhamma Kaninde*, ${ }^{2}$ Robert McGrath, ${ }^{3}$ Mahmoud Belnur, ${ }^{4}$ Naomi McCallion, ${ }^{5}$ Fiona Browne, ${ }^{6}$ Robert Cunney. ${ }^{7}$ Dept. of Neonatology, The Rotunda Hospital , Dublin, Ireland; ${ }^{2}$ Dept. of Neonatology, The Rotunda Hospital, Dublin , Ireland; ${ }^{3}$ Dept.of Neonatology, The Rotunda Hospital , Dublin, Ireland; ${ }^{4}$ Dept. of Neonatology, The Rotunda Hospital, Dublin, Ireland; ${ }^{5}$ Dept. of Dermatology, Children's University Hospital, Templestreet, Dublin, Ireland; ${ }^{6}$ Dept. of Microbiology, Children's University Hospital, Templestreet, Dublin, Ireland

10.1136/archdischild-2019-epa.821

Background Cutaneous fungal infections in healthy neonates are a very rare occurrence. We report a case of a previously well seventeen-day-old neonate presenting with a five-day history of multiple annular skin lesions. These were located on the neck, groin and scalp. Microbiological culture of skin scrapings confirmed the presence of trichophyton soudanense. Trichophyton soudanense is an anthrophilic dermatophyte endemic to sub-Saharan Africa, however it is now recognised as an emerging cause of dermatophytoses in the Western world - in our case Tinea Capitis and Tinea Corporis.

Case Summary A seventeen-day-old neonate presented to our paediatric outpatient department with an annular skin rash. Five days previously lesions had been noted on the scalp and over subsequent days similar lesions appeared on the neck and groin. There were no associated symptoms and the baby was afebrile and had been feeding well. The patient's mother was an African lady from Sierra Leone who had traveled to Ireland while pregnant at 35 weeks gestation. The baby was born at 40 weeks gestation via spontaneous vaginal delivery. The mother developed intra-partum pyrexia, investigations for sepsis were performed and she was commenced on intravenous antibiotics. The high vaginal swab was positive for Group B streptococcus and maternal CRP was $105 \mathrm{mg} / \mathrm{L}$. In light of the maternal history had a workup for sepsis performed and was commenced on intravenous antibiotics. Investigations were negative, antibiotics were discontinued and the baby was clinically well on discharge from the hospital. On routine attendance at POPD five days post delivery, the baby was clinically well with no evident skin lesions.

The patient was referred to the POPD from his primary care provider. An annular rash was noted at the base of the neck, measuring $1.5 \mathrm{~cm}$ in diameter with central clearing. Similar lesions were noted on the scalp - which had associated circumferential pustular lesions, and in the groin - which had an erythematous base. Skin scrapings were sent for microbiological investigation.

The skin scraping was incubated on potato dextrose and dermasel agar at $30^{\circ} \mathrm{C}$. At five days isolates were identified based on distinctive colony morphology i.e. fluffy yellow colour growth and microscopic features as Trichophyton soudanense.
Follow up Skin rash disappeared in ten days with residual hyperpigmentation which is typically seen in Trichophyton infections.

Conclusion Though extremely rare, ringworm or tinea infection should be considered as differential diagnosis while dealing with annular lesions

\section{P486 AUDIT OF PROPHYLACTIC VITAMIN K DOCUMENTATION IN UNIVERSITY MATERNITY HOSPITAL LIMERICK}

Aisha ljaz*, Mohamed Mohamed, Mohammed Asghar, Rizwan Khan, Mohammed Zia, Niazy Al Asaaf. University Maternity Hospital, Limerick, Ireland

\subsection{6/archdischild-2019-epa.822}

Background Phytomenadione (Vitamin K1) is given at birth to all neonates for the prevention of Vitamin K Deficiency Bleeding (VKDB) because there is decline in vitamin $\mathrm{k}$ after birth. Reasons for decline are lack of intestinal bacteria required to synthesize vitamin $\mathrm{K}$, quick depletion in stores after birth, poor vitamin $\mathrm{k}$ transfer across placenta and low vitamin $\mathrm{k}$ concentration in breast milk.

Objectives To evaluate adherence to the policy for administration of Phytomenadione (Vitamin k) to Neonates and documentation of prophylactic vitamin $\mathrm{k}$, in UMHL.

Methodology This is a Prospective study, conducted in University Maternity Hospital Limerick, Limerick, Ireland, in postnatal wards and Neonatal Intensive Care Unit (NICU), during the period from $01 / 08 / 2017$ to $31 / 08 / 2017$ with exclusion criteria of not including babies born outside hospital

Results Total of 50 patients Healthcare records were assessed, 32 in postnatal wards and 18 in NICU. 48(96\%) have documented evidence of vitamin $\mathrm{K}$ injection. 48 (96\%) mothers consented for vitamin $\mathrm{k}$ administration; in 29(60\%) there was evidence of verbal consent compared to 19 with no evidence of consent. On PNW in $90 \%$ prescription date, dose and route were mentioned and $40 \%$ parents received information leaflets. In NICU there was 100\% documentation of date, dose and route of administration while no parents were give information leaflets.

Conclusion There was a good compliance to the audited policy, in term of number of patients who received vit $k$ but there was a significant deficiency in documentation practice and information giving to parents.

\section{P487 CHANGING PATTERNS IN PRESSOR SUPPORT IN THE NEONATAL INTENSIVE CARE UNIT. A TEN-YEAR OVERVIEW}

Caitríona Ní Chathasaigh*, Montse Corderroura, Prof John. F Murphy. The National Maternity Hospital, Holles St, Dublin, Ireland

\subsection{6/archdischild-2019-epa.823}

Background \& Aims Haemodynamic instability is a problem frequently encountered in Neonatal Intensive Care. It is a relatively common complication of preterm birth. In term neonates, it is often associated with significant disease processes such as pulmonary hypertension and sepsis. Infants with low blood pressure are usually treated with fluids, inotropes and occasionally steroids, in cases of refractory hypotension. The 